\title{
Avaliação do Conhecimento de Idosos sobre HIV/AIDS
}

Evaluation the Elderly's Knowledge about HIV / AIDS

Evaluación del conocimiento de los ancianos sobre el VIH/SIDA

Liliane Cristina BARBOSA Departamento de Odontologia Preventiva e Restauradora, Faculdade de Odontologia, UNESP Univ. Estadual Paulista, 16015-050 Araçatuba - SP, Brasil https://orcid.org/0000-0001-8776-6401 Tânia Adas SALIBA Departamento de Odontologia Preventiva e Restauradora, Faculdade de Odontologia, UNESP Univ. Estadual Paulista, 16015-050 Araçatuba - SP, Brasil

https://orcid.org/0000-0003-1327-2913

Artênio José Ísper GARBIN

Departamento de Odontologia Preventiva e Restauradora, Faculdade de Odontologia, UNESP Univ. Estadual Paulista, 16015-050 Araçatuba - SP, Brasil

https://orcid.org/0000-0002-7017-8942

Cléa Adas Saliba GARBIN

Departamento de Odontologia Preventiva e Restauradora, Faculdade de Odontologia, UNESP Univ. Estadual Paulista, 16015-050 Araçatuba - SP, Brasil

https://orcid.org/0000-0001-5069-8812

\section{Resumo}

Trata-se de um estudo transversal com 61 idosos frequentadores de uma associação promotora de atividade de lazer vinculada a órgão público em um município do Estado de São Paulo, cujo objetivo foi avaliar o conhecimento do idoso em relação ao HIV/Aids. Os dados foram coletados por meio de questionário aplicado por um único pesquisador. Dentre os investigados, $64 \%$ era do sexo feminino; a faixa etária predominante (50,8\%) entre 60 e 69 anos e 55,7\% não estavam vivendo com o companheiro. Do total, $56 \%$ possuía vida sexual ativa, porém $73,5 \%$ afirmaram não usar preservativos durante a relação sexual. Observou-se maior atividade sexual para o gênero masculino $(81,8 \%)$ e entre os participantes que viviam com seus companheiros (92,6\%). O não uso do preservativo esteve associado ao gênero feminino (93,8\%) e ao fato de viver com o companheiro (88\%). Quanto à transmissão do HIV, ainda persiste uma parcela que acredita que beijo na boca (34\%); compartilhamento de copos e talheres (18\%); uso do mesmo sanitário (25\%) e picada de mosquito (30\%) são meios de transmissão da doença. Os resultados demonstraram lacunas no conhecimento dos idosos quanto à forma de transmissão e prevenção do HIV, o que remete à vulnerabilidade e desafia a saúde pública, sendo necessário pensar em políticas de criação ou reformulação de ações em saúde para os idosos, visando, além do preenchimento destas lacunas, a contribuição para mudanças de comportamento e adoção de prática sexual segura.

Descritores: Idoso; Doenças Sexualmente Transmissíveis; Epidemiologia.

\section{Abstract}

This is a cross-sectional study with 61 elderly people who attend a leisure promotion association linked to a public agency in a city in the state of São Paulo, whose objective was to evaluate the elderly's knowledge about HIV / AIDS. Data were collected through a questionnaire administered by a single researcher. Among the investigated, $64 \%$ were female; the predominant age group (50.8\%) between 60 and 69 years and 55.7\% were not living with their partner. Of the total, $56 \%$ had an active sex life, but $73.5 \%$ said they did not use condoms during sexual intercourse. Higher sexual activity was observed for males (81.8\%) and among participants who lived with their partners (92.6\%). Not using condoms was associated with female gender (93.8\%) and living with a partner (88\%). As for HIV transmission, there is still a portion that believes kiss on the mouth (34\%); sharing glasses and cutlery (18\%); use of the same toilet (25\%) and mosquito bites (30\%) are means of disease transmission. The results showed gaps in the knowledge of the elderly about the way of transmission and prevention of HIV, which refers to vulnerability and challenges public health, being necessary to think about policies for the creation or reformulation of health actions for the elderly, aiming, in addition to filling these gaps, contributing to behavior change and adopting safe sexual practice.

Descriptors: Aged; Sexually Transmitted Diseases; Epidemiology.

\section{Resumen}

Se trata de un estudio transversal con 61 ancianos asistentes a una asociación promotora de actividades de ocio vinculada a una agencia pública de un municipio del Estado de São Paulo, cuyo objetivo fue evaluar el conocimiento de los ancianos en relación al VIH/SIDA. Los datos fueron recolectados a través de un cuestionario aplicado por un solo investigador. Entre los investigados, el $64 \%$ eran mujeres; el grupo de edad predominante $(50,8 \%)$ estaba entre 60 y 69 años y el $55,7 \%$ no vivía en pareja. Del total, el $56 \%$ tenía vida sexual activa, pero el $73,5 \%$ dijo no usar preservativo durante las relaciones sexuales. Se observó más actividad sexual para los hombres (81,8\%) y entre los participantes que vivían con sus parejas (92,6\%). El no uso de preservativo se asoció al género femenino $(93,8 \%)$ y al hecho de vivir en pareja $(88 \%)$. En cuanto a la transmisión del VIH, todavía hay una porción que cree que los besos en la boca (34\%); compartir vasos y cubiertos (18\%); el uso del mismo baño $(25 \%)$ y las picaduras de mosquitos $(30 \%)$ son medios de transmisión de la enfermedad. Los resultados mostraron lagunas en el conocimiento de los ancianos sobre la forma de transmisión y prevención del VIH, lo que se refiere a la vulnerabilidad y desafíos a la salud pública, lo que hace necesario pensar en políticas para crear o reformular acciones de salud para los ancianos, con el objetivo, además al llenado de estos vacíos, contribuyendo a cambios de comportamiento y adopción de prácticas sexuales seguras.

Descriptores: Anciano; Enfermedades de Transmisión Sexual; Epidemiología.

INTRODUÇÃO

Por muito tempo entendia-se a pessoa
idosa como alguém doente, incapaz,
improdutiva e dependente da família e da
comunidade em geral. Assistimos às mudanças
deste antigo conceito para uma nova
denominação de envelhecimento saudável, que

está associado à promoção do bem-estar físico, psíquico e social, por meio de estratégias voltadas ao convívio, interação, inclusão e participação social do idoso'.

O Brasil conta, hoje, com mais de 12 milhões de pessoas com idade acima de 60 anos, representando aproximadamente $12 \%$ da 
população em geral, com estimativas de aumento para $28 \%$ em $2060^{2}$.

O crescimento quantitativo da população idosa, aliado ao aumento da expectativa e qualidade de vida e aos avanços tecnológicos em saúde, como os tratamentos de reposição hormonal e medicações para impotência, principalmente o Sildenafil (Viagra(C), têm permitido 0 redescobrimento de novas experiências, como o sexo entre os idosos ${ }^{3-5}$. Todavia, as práticas sexuais inseguras tornam essa população vulnerável às infecções pelo Vírus da Imunodeficiência Humana (HIV) e a outras doenças sexualmente transmissíveis (DST), como a sífilis, clamídia e gonorreia ${ }^{6}$.

Estudos mostram que apesar do desempenho sexual dos idosos ser beneficiado pelos avanços científicos e tecnológicos, eles ainda acreditam que as infecções sexualmente transmissíveis (IST) são prováveis apenas em pessoas que levam uma vida promíscua. Esse ceticismo, juntamente com a resistência ao uso do preservativo, por considerá-lo apenas um método contraceptivo, torna essa população ainda mais vulnerável ${ }^{6-10}$.

A transição demográfica $\mathrm{e}$ epidemiológica da população lança um desafio para a sociedade em geral em lidar com 0 processo de envelhecimento. Quando se trata das políticas públicas de promoção da saúde no contexto das DST, observa-se que estão voltadas para populações mais jovens e àquelas reconhecidas como mais vulneráveis. Pensar que a população idosa é capaz de manter suas relações sexuais e sentir prazer, mesmo com as alterações fisiológicas decorrentes do envelhecimento, contribui para o fortalecimento de estratégias voltadas à saúde sexual responsável para homens e mulheres ${ }^{11-15}$.

Os estereótipos relacionados aos adultos mais velhos como assexuados ou menos sexuais, podem causar constrangimento pessoal e preocupações, ainda assim, quando se olha para a população idosa como sexualmente ativa, o enfoque contempla apenas casais heterossexuais e monogâmicos ${ }^{16-19}$.

O aumento da incidência de HIV na população acima dos 60 anos é um problema de saúde pública. Este acréscimo está diretamente associado ao avanço das tecnologias de diagnóstico e a terapia antirretroviral (TARV). Assim, à medida que aumenta 0 acesso ao tratamento, aumenta também a sobrevida das pessoas com HIV e isto faz que muitas ultrapassem os 60 anos de idade $^{16,20}$.

Existe, portanto, a necessidade de conhecer as mudanças comportamentais desta população e pesquisar sobre o tema com o intuito de contribuir para a compreensão da sexualidade do idoso e estimular o desenvolvimento de políticas públicas voltadas para a saúde sexual e a prevenção às IST/HIV/AIDS.

Diante deste fato, o presente estudo objetiva avaliar o conhecimento sobre o modo de transmissão, prevenção e proteção das doenças sexualmente transmissíveis e estimar a proporção sexualmente ativa entre os idosos.

MATERIAL E MÉTODO

Trata-se de estudo transversal, descritivo e quantitativo com idosos participantes de uma associação promotora de atividade de lazer vinculada a órgão público em um município do Estado de São Paulo. Essa Associação realiza bailes aos sábados e a coleta ocorreu nos meses de maio e junho de 2019, em frente à porta do clube, antes do início de cada evento. O local para a coleta de dados foi selecionado devido ao fato de ser um clube específico para a realização dos bailes da terceira idade. Participaram do estudo as pessoas com idade de 60 anos ou mais que voluntariamente responderam ao questionário, finalizando a amostragem, quando todos os frequentadores já haviam sido convidados.

A coleta de dados, foi do tipo inquérito, por meio de questionário com questões fechadas, compreendendo a caracterização do perfil sociodemográfico, conhecimento e a percepção, dos idosos, acerca das formas de prevenção, proteção e transmissão das IST/HIV/aids. A duração do inquérito foi de aproximadamente 10 minutos, realizado por um pesquisador treinado. Estudo piloto foi realizado para adequação do instrumento, sendo estes participantes excluídos da amostra. A amostra final foi composta por 61 idosos. Os dados foram processados no programa Epi Info versão 7.2 e analisados no BioEstat versão 5.3. Foram realizadas análises descritivas exploratórias dos dados a partir da apuração de frequências simples absolutas e percentuais e para avaliar a associação entre o perfil dos participantes e a atividade sexual aplicou-se teste qui-quadrado ou exato de Fisher, após dicotomização das variáveis categóricas.

Atendendo às recomendações da Resolução no 466/12 do Conselho Nacional de Saúde, o estudo foi aprovado pelo Comitê de Ética em Pesquisa da Universidade Estadual Paulista, por meio do parecer no: 3.326.491. Todos os participantes concordaram e assinaram o termo de consentimento livre e esclarecido. 
RESULTADOS

Participaram da pesquisa 61 idosos, sendo $64 \%$ do sexo feminino. A faixa etária entre 60 e 69 anos foi predominante $(50,8 \%)$ e $55,7 \%$ não estavam vivendo com o companheiro. Em relação à escolaridade, 13,1\% da amostra alcançou nível superior, enquanto que $42,6 \%$ não concluiu o ensino fundamental e a religião a católica correspondeu a $83,6 \%$ dos entrevistados (Tabela 1).

Tabela 1: Descrição da amostra do estudo $(n=61)$ em llha Solteira, SP.

\begin{tabular}{l|c|c}
\hline Sexo & $\mathbf{n}$ & $\mathbf{\%}$ \\
\hline Masculino & $\mathbf{2 2}$ & 36,0 \\
\hline Feminino & 39 & 64,0 \\
\hline Idade & 31 & 50,8 \\
\hline 60 a 69 anos & 23 & 37,7 \\
\hline 70 a 79 anos & 7 & 11,5 \\
\hline$>80$ anos & 2 & \\
\hline Estado civil: & 20 & 32,3 \\
\hline Solteiro (a) & 24 & 39,3 \\
\hline Divorciado (a) & 15 & 24,6 \\
\hline Viúvo (a) & & \\
\hline Casado (a) & 27 & 44,3 \\
\hline Vive com o companheiro (a) & 34 & 55,7 \\
\hline Sim & & \\
\hline Não & 26 & 42,6 \\
\hline Escolaridade & 13 & $\mathbf{2 1 , 3}$ \\
\hline Fundamental incompleto & 10 & 16,4 \\
\hline Fundamental completo & 4 & 6,6 \\
\hline Ensino médio completo & 8 & 13,1 \\
\hline Superior incompleto & & \\
\hline Superior completo & 2 & 3,3 \\
\hline Religião & 51 & 83,6 \\
\hline Nenhuma & 3 & 4,9 \\
\hline Católica & 4 & 6,6 \\
\hline Evangélica & 1 & 1,6 \\
\hline Espírita & & \\
\hline Outra & & \\
\hline
\end{tabular}

\section{- Percepção sobre doenças sexualmente transmissíveis}

Dos 61 entrevistados, 56\% tinha vida sexual ativa à época da entrevista, ou seja mantiveram relação sexual nos últimos seis meses. Destes, 98\% concordou que a camisinha previne a transmissão do vírus HIV e das IST, apesar de $73,5 \%$ não utilizar a camisinha durante a relação sexual. Quanto à realização do teste da aids a porcentagem dos idosos que relatou ter feito o teste foi de $43 \%$, e os que pretendem fazer o teste futuramente foi de $39 \%$. Os participantes acreditam na possibilidade de estar com alguma IST caso aparecesse feridas (89\%), verrugas (82\%) e corrimento $(69 \%)$, nos órgãos genitais.

- Comportamento dos participantes sexualmente ativos

Dos idosos com vida sexual ativa $(n=34)$ à época da entrevista, 73,5\% afirmou não usar preservativo durante a relação sexual; $88,2 \%$ relatou ter parceiro fixo e $97 \%$ respondeu que nos últimos seis meses não tiveram relações sexuais com mais de seis parceiros. Todos estes afirmaram que nos últimos seis meses não tiveram nenhum tipo de doença sexualmente transmissível e não procuraram os serviços de saúde por causa de aparecimento de feridas ou corrimentos nos órgãos genitais.
A tabela 2 apresenta os resultados das análises univariadas entre as características dos idosos e a atividade sexual nos últimos seis meses. Foram selecionadas as seguintes variáveis: gênero, idade, se vive com o companheiro, grau de instrução e religião. Quando analisada a variável 'gênero', houve significância estatística $(p=0,002)$, observandose maior atividade sexual para o gênero masculino em relação ao feminino. Com a variável 'vive com o companheiro', também estatisticamente significante $(p=0,000)$ foi observado que os participantes que vivem com seus companheiros estão mais sexualmente ativos quando comparados aos que não estão vivendo com seus companheiros.

Tabela 2. Análise univariada do perfil dos participantes associado à atividade sexual, Ilha Solteira, SP, 2019. $(n=61)$

\begin{tabular}{|c|c|c|c|}
\hline Características & $\begin{array}{c}\begin{array}{c}\text { Sexualmente } \\
\text { ativo }\end{array} \\
\text { n (\%) }\end{array}$ & $\begin{array}{c}\begin{array}{c}\text { Não sexualmente } \\
\text { ativo }\end{array} \\
\text { n (\%) } \\
\end{array}$ & $\mathbf{p}$ \\
\hline \multicolumn{4}{|l|}{ Sexo } \\
\hline Feminino & $16(41,0)$ & $23(59,0)$ & \multirow[b]{2}{*}{ o, $002^{*}$} \\
\hline Masculino & $18(81,8)$ & $4(18,2)$ & \\
\hline \multicolumn{4}{|l|}{ Idade } \\
\hline 60 a 69 anos & $18(58,0)$ & $13(42,0)$ & \multirow{2}{*}{0,709} \\
\hline 70 anos ou mais & $16(53,3)$ & $14(46,7)$ & \\
\hline \multicolumn{4}{|c|}{ Vive com o companheiro } \\
\hline Sim & $25(92,8)$ & $2(7,4)$ & \multirow{2}{*}{$\mathbf{0 , \mathbf { O O o } ^ { * }}$} \\
\hline Não & $9(26,5)$ & $25(73,5)$ & \\
\hline \multicolumn{4}{|l|}{ Grau de instrução } \\
\hline Até 7 anos de estudo & $16(61,5)$ & $10(38,5)$ & \multirow{2}{*}{0,431} \\
\hline 8 anos ou mais & $18(51,4)$ & $17(48,6)$ & \\
\hline \multicolumn{4}{|l|}{ Religião } \\
\hline Católica & $30(58,8)$ & $21(41,2)$ & \multirow[b]{2}{*}{0,226} \\
\hline Outra & $4(40,0)$ & $6(60,0)$ & \\
\hline
\end{tabular}

Para os idosos sexualmente ativos, a tabela 3 apresenta os resultados das análises univariadas entre as características dos idosos e o uso de preservativo durante as relações. Foram selecionadas as variáveis: gênero, idade, se vive com o companheiro, grau de instrução e religião. Houve significância estatística para a variável 'gênero' $(p=0,014)$, observando-se que a resistência em usar o preservativo foi maior no gênero feminino em relação ao masculino. Quanto ao fato de estar vivendo com o companheiro, a variável também apresentou significância estatística $(p=0,003)$, na qual foi observado que dos participantes que vivem com seus companheiros, em $88 \%$ dos casos eles não utilizam preservativo durante as relações sexuais.

- Conhecimento sobre as formas de transmissão do HIV

A maioria (98\%) dos idosos concorda que - sexo sem camisinha representa risco de contrair a doença, entretanto, ainda há uma parcela que acredita que beijo na boca, compartilhar copos e talheres, que o uso do mesmo sanitário e que picada de mosquito são meios de transmissão (Gráfico 1). Ainda em relação as formas de transmissão do HIV, não houve associação estatisticamente significante 
com as variáveis gênero, idade, tempo de estudo e religião, conforme apresentado na tabela 4.

Tabela 3. Análise univariada do perfil dos participantes sexualmente ativos associado ao uso de preservativo nas relações sexuais, Ilha Solteira, SP, 2019. $(n=34)$

\begin{tabular}{|c|c|c|c|}
\hline \multirow[b]{2}{*}{ Características } & Usa preservativo & \multirow{2}{*}{$\begin{array}{c}\text { Não usa preservativo } \\
\text { n (\%) }\end{array}$} & \multirow[b]{2}{*}{$\mathbf{p}$} \\
\hline & n (\%) & & \\
\hline \multicolumn{4}{|l|}{ Sexo } \\
\hline Feminino & $1(6,2)$ & $15(93,8)$ & \\
\hline Masculino & $8(44,4)$ & $10(55,6)$ & $\mathbf{0 , 0 1 4}$ * \\
\hline \multicolumn{4}{|l|}{ Idade } \\
\hline 60 a 69 anos & $6(33,3)$ & $12(66,7)$ & \multirow{2}{*}{$0,285^{*}$} \\
\hline 70 anos ou mais & $3(18,8)$ & $13(81,2)$ & \\
\hline \multicolumn{4}{|c|}{ Vive com o companheiro } \\
\hline $\begin{array}{l}\text { Sim } \\
\text { Não }\end{array}$ & $3(12,0)$ & $22(88,0)$ & $\mathbf{0 , 0 0 3}{ }^{*}$ \\
\hline \multicolumn{4}{|c|}{ Grau de instrução } \\
\hline $\begin{array}{l}\text { Até } 7 \text { anos de } \\
\text { estudo }\end{array}$ & & $12(75,0)$ & \multirow[t]{2}{*}{$0,582^{*}$} \\
\hline 8 anos ou mais & $5(27,8)$ & $13(72,2)$ & \\
\hline \multicolumn{4}{|l|}{ Religião } \\
\hline Católica & $8(26,7)$ & $22(73,3)$ & \multirow{2}{*}{$0,719^{*}$} \\
\hline Outra & $1(25,0)$ & $3(75,0)$ & \\
\hline
\end{tabular}

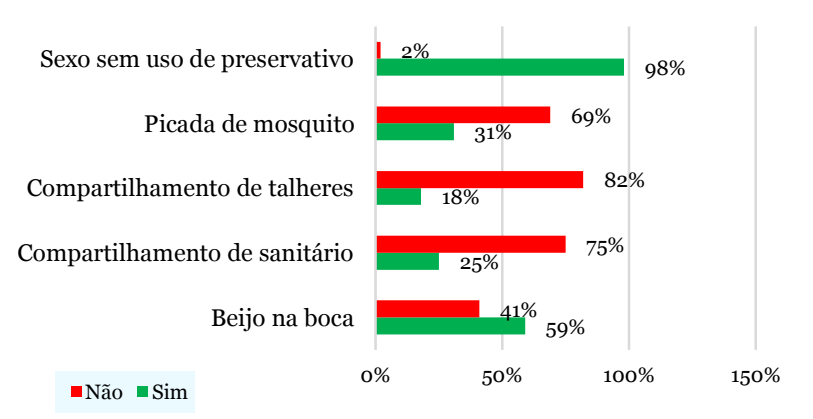

Gráfico 1: Distribuição da percepção dos idosos sobre as formas de transmissão do HIV. Ilha Solteira - SP, 2019.

Tabela 4: Análise univariada dos fatores associados às respostas afirmativas sobre as formas de transmissão do HIV, Ilha Solteira, SP, 2019. $(\mathrm{n}=61)$

\begin{tabular}{|c|c|c|c|c|c|}
\hline & Total & $\begin{array}{c}\text { Talheres } \\
\text { n (\%) }\end{array}$ & $\begin{array}{c}\text { Banheiro } \\
\text { n (\%) }\end{array}$ & $\begin{array}{c}\text { Beijo na boca } \\
\text { n (\%) }\end{array}$ & $\begin{array}{c}\text { Mosquito } \\
\text { n (\%) }\end{array}$ \\
\hline Gênero & & $\mathrm{p}=0,381$ & $\mathrm{p}=0,117$ & $\mathrm{p}=0,593$ & $\mathrm{p}=0,085$ \\
\hline Feminino & 39 & $8(20,5)$ & $12(30,7)$ & $24(61,5)$ & $15(38,4)$ \\
\hline Masculino & 22 & $3(13,6)$ & $3(13,6)$ & $12(54,5)$ & $4(18,2)$ \\
\hline Idade & & $\mathrm{p}=0,739$ & $\mathrm{p}=0,822$ & $\mathrm{p}=0,232$ & $\mathrm{p}=0,142$ \\
\hline 60 a 69 anos & 31 & $5(16,1)$ & $8(25,8)$ & $16(51,6)$ & $7(22,5)$ \\
\hline $\begin{array}{l}70 \text { anos ou } \\
\text { mais }\end{array}$ & 30 & $6(20,0)$ & $7(23,3)$ & $20(66,6)$ & $12(40,0)$ \\
\hline $\begin{array}{l}\text { Anos de } \\
\text { estudo }\end{array}$ & & $\mathrm{p}=\mathbf{0}, 454$ & $\mathrm{p}=0,334$ & $\mathrm{p}=0,856$ & $\mathrm{p}=0,287$ \\
\hline Até 7 anos & 26 & $4(15,4)$ & $8(30,7)$ & $15(57,7)$ & $10(38,4)$ \\
\hline $\begin{array}{l}8 \text { anos ou } \\
\text { mais }\end{array}$ & 35 & $7(20,0)$ & $7(20,0)$ & $21(60,0)$ & $9(25,7)$ \\
\hline Religião & & $\mathrm{p}=0,419$ & $\mathrm{p}=0,056$ & $\mathrm{p}=0,384$ & $\mathrm{p}=0,624$ \\
\hline Católica & 51 & $10(19,6)$ & $10(19,6)$ & $31(60,7)$ & $16(31,4)$ \\
\hline Outra & 10 & $1(10,0)$ & $5(50,0)$ & $5(50,0)$ & $3(30,0)$ \\
\hline
\end{tabular}

${ }^{*}$ Estatisticamente significante à $p<0,05$

DISCUSSÃO

O prolongamento da vida sexual dos idosos associado à ocorrência de práticas inseguras têm contribuído com o aumento da vulnerabilidade às doenças sexualmente transmissíveis, entre elas a Síndrome da Imunodeficiência Adquirida ${ }^{3,4}$.

A predominância das mulheres na população idosa caracteriza a feminização do envelhecimento, observado em diversos estudos. A sobrevida das mulheres é de aproximadamente cinco anos a mais que dos homens, visto que elas apresentam uma maior preocupação com a prevenção de doenças, adotando uma rotina de exames para acompanhamento e manutenção da saúde s0,21,22. $^{10}$.

Sabe-se que o grau de instrução é um indicador não só do nível socioeconômico mas também de impacto na saúde. A escolaridade influencia na apropriação de informações, sendo que as pessoas com escolaridade mais elevada possuem mais formas de acesso à informação, como livros, internet e revistas, além de também possuírem uma capacidade de assimilação maior do que aqueles que não possuem ${ }^{14,21}$.

A literatura confirma os resultados obtidos neste estudo, que o nível de conhecimento dos idosos sobre as DST ou até mesmo o uso de preservativos pelos mesmos, não apresenta associação significativa com as variáveis estudadas ${ }^{14,21-23}$. Os resultados mostraram que os idosos estão, cada vez mais sexualmente ativos, no entanto resistentes quanto ao uso de preservativos ${ }^{21-23}$.

O percentual de mulheres sexualmente ativas é expressivo, em virtude dos avanços científicos e tecnológicos, como o caso da reposição hormonal. Estudos mostram que a disfunção sexual feminina, em sua maioria, não é de ordem orgânica, mas sim psíquica, causada por estresse, depressão, fadiga, ansiedade e ou vergonha ${ }^{24}$.

A resistência ao uso do preservativo ainda persiste e pode estar relacionada ao fato de considerá-lo apenas um método contraceptivo, ou ainda estar relacionada à incapacidade de negociarem 0 uso de preservativo nas relações. A estabilidade do relacionamento e submissão ao companheiro representam barreiras impeditivas ao uso de preservativos $^{25}$. É necessário desenvolver atividades educativas com mulheres, de forma que se sintam em condições de exigirem o uso de preservativos para a prática de sexo seguro $^{26}$.

A percepção sobre a transmissão do vírus pelo beijo na boca, pelo uso compartilhado de sanitários, copos e talheres foi alta, diferentemente de outros estudos, nos quais essas percepções foram reduzidas, somente após intervenções. Ratifica-se assim, a necessidade de políticas públicas que desmistifiquem ideias errôneas e esclareçam a população idosa sobre a transmissibilidade do vírus do $\mathrm{HIV}^{22,23}$.

Ainda há aqueles que acreditam que o mosquito é um vetor na transmissão do HIV. Urge esclarecer que o mosquito não tem como ser um vetor para a transmissão do HIV, devido à baixa infectividade e ao curto período de sobrevivência do vírus no organismo do mosquito $^{10,27}$. 
Os casos de Aids têm aumentado entre os idosos, que até bem pouco tempo representavam pequeno percentual estatístico da doença. No Brasil, os casos de aids na faixa etária acima de 60 anos passou de 10,3\% em 2007 para 13,4 \% em 2017 nos homens e de $5,3 \%$ em 2007 para 10,3\% em 2017 entre as mulheres. No geral $2,5 \%$ dos idosos são portadores do HIV e esse percentual pode ser superior, levando-se em conta a subnotificação ${ }^{28}$.

Observa-se uma carência na literatura em relação aos estudos de prevalência das doenças sexualmente transmissíveis na população idosa, bem como o conhecimento que esse grupo tem sobre tais doenças e como deve ser 0 tratamento adequado nas diferentes situações. Os esforços, na grande maioria, concentram-se na notificação de casos do HIV/AIDS e no tratamento por meio dos medicamentos antirretrovirais ${ }^{9,26}$.

CONCLUSÃO

Apesar do bom nível de informação entre os idosos, surgiram lacunas quanto à forma de transmissão e prevenção do HIV, fato que remete à vulnerabilidade dessa população e desafia a saúde pública. É necessário, portanto, pensar em políticas de criação ou reformulação de ações em saúde para os idosos, visando, além do preenchimento destas lacunas, a contribuição para mudanças de comportamento e adoção de prática sexual segura.

\section{REFERENCIAS}

1. Antunes MCP, Moreira MC. Educação intergeracional e envelhecimento bemsucedido. RBCEH. 2018;15(1):21-32.

2. IBGE. Instituto Brasileiro de Geografia e Estatística. Projeção da população do Brasil por sexo e idade 2010-2060: Revisão 2008. Disponível em: <https://www.ibge.gov.br/estatisticas/sociais/po pulacao/9109-projecao-da-populacao> Acesso em 12 abr. 2021.

3. Brasil. Ministério da Saúde. Secretaria de Atenção à Saúde. Departamento de Ações Programáticas e Estratégicas. Atenção à saúde da pessoa idosa e envelhecimento. Brasília: 2010.

https://bvsms.saude.gov.br/bvs/publicacoes/ate ncao_saude_pessoa_idosa_envelhecimento_v 12.pdf

4. Batista AFDO, Marques APDO, Leal MCC, Marino JG, Melo HMDA. Idosos: associação entre o conhecimento da aids, atividade sexual e condições sociodemográficas. Rev Bras Geriatr Gerontol. 2011;14(1):39-48.

5. Rodrigues LR, Portilho P, Tieppo A, Chambo A. Análise do comportamento sexual de idosas atendidas em um ambulatório de ginecologia. Rev Bras Geriatr Gerontol. 2018;21(6):724-30.

6. Laroque MF, Affeldt ÂB, Cardoso DH, Souza GLD, Santana MDG, Lange C. Sexualidade do idoso: comportamento para a prevenção de DST/AIDS. Rev Gaúch Enferm. 2011;32: 774-80.

7. Bienko M. Promotion of sexual health among seniors in the self-help media realm of popular culture. Anthropological Review. 2015;78(3): 251-57.

8. Oliveira MLC, Paz LC, Melo GF. Ten years of HIV-AIDS epidemic in more than 60 years in Federal District - Brazil. Rev Bras Epidemiol. 2013;16(1):30-9.

9. Dornelas Neto J, Nakamura AS, Cortez LE, Yamaguchi MU. Doenças sexualmente transmissíveis em idosos: uma revisão sistemática [Sexually transmitted diseases among the elderly: a systematic review]. Cien Saude Colet. 2015;20(12):3853-64.

10. Nascimento RG, Monteiro EL, Ferreira LS, Santos ZL. Nível de conhecimento de idosos comunitários em relação ao HIV/Aids: estudo exploratório na rede básica de saúde de Belém, Pará, Brasil. RBCEH. 2013;10(1):113-22.

11. Freitas CASL, da Silva MJ, Brito MDCC, Braga VAB, Bessa MEP. Health policies for the elderly and their families: na integrative literature review. Rev enferm UFPE on line.. 2011;5(9):2300-8.

12. Garcia GS, Lima LF, Silva JB, Andrade LD, Abrão FMS. Vulnerabilidade dos idosos frente ao HIV/aids: tendências da produção científica atual no Brasil. J Bras Doenças Sex Transm. 2012; 24(3):183-8.

13. Bittencourt GKGD, Kamylla F, Meira LCS, Santos MCF, SILVA A. Concepções de idosos sobre vulnerabilidades ao HIV/AIDS. Riase online. 2016;2(1):407-20.

14. De Brito NMI, da Costa Andrade SS, da Silva FMC, Fernandes MRCC, Brito KKG, dos Santos Oliveira SH. Elderly, sexually tranmistted infections and aids: Knowledge and risk perception. ABCS Health Sciences. 2016;41(3):140-45.

15. Haesler E, Bauer M, Fetherstonhaugh D. Sexuality, sexual health and older people: a systematic review of research on the knowledge and attitudes of health professionals. Nurse Educ Today.2016;40:57-71.

16. Santos A, Assis M. Vulnerabilidade das idosas ao HIV/AIDS: despertar das políticas públicas e profissionais de saúde no contexto da atenção integral: revisão de literatura. Rev Bras Geriatr Gerontol.2011;14(1):147-57.

17. Brasil. Ministério da Saúde. Boletim epidemiológico: aids e DST. Brasília, ano V, n.1, 2017. http://www.aids.gov.br/pt-br/centraisde-conteudos/boletins-epidemiologicos-vertical 
18. Cordeiro LI, Lopes TDO, Lira LEDA, Feitoza SMDS, Bessa MEP, Pereira MLD, Feitoza AR, Souza ARD. Validation of educational booklet for HIV/Aids prevention in older adults. Rev Bras Enferm. 2017;70:775-82.

19. Srinivasan S, Glover J, Tampi RR, Tampi DJ, Sewell DD. Sexuality and the Older Adult. Curr Psychiatry Rep. 2019;21(10):97.

20. Aguiar RB, Leal MCC, Marques APO. Knowledge and attitudes about sexuality in the elderly with HIV. Cien Saude Colet. 2020;25(6): 2051-62.

21. Nardelli GG, Malaquias BSS, Gaudenci EM, Ledic CS, Azevedo NF, Martins VE, Santos ÁDS. Knowledge about the human immunodeficiency syndrome among elders in a unit for the care of the elderly. Rev Gaúch Enferm. 2017;37(spe):e2016-0039.

22. Bastos LM, Tolentino JMS, Frota MADO, Tomaz WC, Fialho MLDS, Batista ACB et al. Avaliação do nível de conhecimento em relação à Aids e sífilis por idosos do interior cearense, Brasil. Ciênc Saúde Colet. 2018;23:2495-502.

23. Lazzarotto AR, Santos VSD, Reichert MT, Quevedo DMD, Fossatti P, Santos GAD, Calvetti PU, Sprinz, E. Oficinas educativas sobre HIV/Aids: uma proposta de intervenção para idosos. Rev Bras Geriatr Gerontol. 2013;16:833-43.

24. Araújo SL, Zazula R. Sexualidade na terceira idade e terapia comportamental: revisão integrativa. RBCEH. 2015;12(2):172-82.

25. Silva CM, Lopes FMVM, Vargens OMC. A vulnerabilidade da mulher idosa em relação à AIDS. Rev Gaúch Enferm. 2010;31(3):450-57.

26. Andrade J, Ayres JA, Alencar RA, Duarte MTC, Parada CMGDL. Vulnerability of the elderly to sexually transmitted infections. Acta Paul Enferm. 2017;30(1):8-15.

27. Iqbal MM. Can we get AIDS from mosquito bites? J LA State Med Soc. 1999;151(8): 429-33.

28. UNAIDS. 90-90-90 An ambitious treatment target to help end the AIDS epidemic. Disponível em: $<$ http://www.unaids.org/en/resources/document s/> Acesso em 20 mai. 2021.

\section{CONFLITO DE INTERESSES}

Os autores declaram não haver conflitos de interesse

AUTOR PARA CORRESPONDÊNCIA

Liliane Cristina Barbosa

Rua José Bonifácio, 1193 - Vila Mendonça

16015-050 Araçatuba - SP, Brasil

E-mail: lilika.bar@gmail.com

Submetido em 09/07/2021

Aceito em 21/08/2021 